\title{
SOLUTION OF A DIFFERENCE EQUATION PERTINENT TO LINEAR, PARAMETRIC ELECTRIC NETWORKS*
}

\author{
By B. J. LEON (Hughes Research Laboratories Malibu, California)
}

This paper presents an algorithm for finding the fundamental system of solutions of a class of linear difference equations relative to a theorem of Perron [1]. This particular fundamental system is important in the analysis of physical systems with periodic parameters such as linear parametric networks [2] and parametric amplifiers. These difference equations arise as the Laplace transforms of the linear differential equations with periodic coefficients that characterize parametric networks. These transforms must be analytic in a half plane. In Ref. [2] it is shown that the method of variation of parameters yields such an analytic transform if the solutions to the homogeneous equation are Perron's fundamental system. These solutions to the homogeneous equations need not be analytic or even continuous.

The homogeneous linear difference equation under consideration is

$$
0=U(\omega+n)+a_{n-1}(\omega) U(\omega+n-1)+\cdots+a_{0}(\omega) U(\omega)
$$

subject to the conditions

$$
\begin{aligned}
& \text { a) } \lim _{\substack{m \rightarrow \infty \\
\text { integer }}} a_{i}(\omega+m)=A_{i}, \text { a constant, } \\
& \text { b) } A_{0} \neq 0 \text {, and } a_{0}(\omega+m) \neq 0 \text { for } m=0,1,2, \cdots, \\
& \text { c) } u^{n}+A_{n-1} u^{n-1}+\cdots+A_{0}=\left(u-u_{1}\right)\left(u-u_{2}\right) \cdots\left(u-u_{n}\right) \\
& \text { d) }\left|u_{i}\right| \neq\left|u_{i}\right| \text { for } i \neq j .
\end{aligned}
$$

In the difference equation literature-Milne-Thomson [3] is the most complete reference for methods of solution - the emphasis is on finding analytic solutions to the homogeneous equation. The complexity of these methods increases markedly with both the order of the difference equation and the complexity of the coefficients of the equation. With these methods, only the simplest of parametric amplifier problems can be put on an IBM 709 computer. If a few second-order effects are included in the amplifier circuit model, the machine overflows.

The algorithm presented in this paper is intended for execution on a digital computer. When the problem is set up on a digital computer, the coefficients in Eq. (1) are subroutines, which are brought into the main program as numbers. The number of operations in the main program depends on the order of the difference equation and on the asymptotic behavior of the coefficients. The complexity of the coefficients for small $\omega$ does not affect the complexity of the main program.

*Received August 12, 1960. 
The basis for our algorithm is the property of the solutions to the difference equation given by Perron's theorem. That theorem states that for Eq. (1) subject to the conditions (2) there exists a fundamental system of solutions, $U_{1}, U_{2}, \cdots, U_{n}$, such that

$$
\lim _{\substack{m \rightarrow \infty \\ \text { integer }}} \frac{U_{i}(\omega+m+1)}{U_{i}(\omega+m)}=u_{i} .
$$

We recall that the definition of a fundamental system of solutions for difference equations is similar to the definition for differential equations except that the arbitrary constants in the differential equation case are replaced by arbitrary periodic functions of period one in $\operatorname{Re}[\omega]$.

The algorithm has five basic computational steps that are repeated over and over until all the desired solutions are found. Let us first describe the steps in words and then present them formally with justification. The steps are

(I) There is a straightforward procedure for constructing solutions to Eq. (1) [4]. Although the solutions so constructed do not have the asymptotic property (3), we may formally write each solution as a linear combination of the members of the desired fundamental system.

(II) From Eq. (3) we see that the asymptotic properties of the desired $U_{i}$ are all different. Thus if we examine solutions generated in step (I) for large values of the argument we can ascertain their relative dependence on the desired $U_{i}$ which has the fastest rate of growth. Call this largest solution $U_{1}$.

(III) Starting from $n$-linearly independent solutions constructed in step (I) with known $U_{1}$ dependence from step (II) we can proceed by systematic elimination to find $(n-1)$ solutions which depend only on $U_{2}, U_{3}, \cdots, U_{n}$.

Since all the $\left|u_{i}\right|$ are distinct, steps (II) and (III) can be repeated eliminating the $U_{2}$ dependence, then $U_{3}$, etc., until there remains only one solution which has the asymptotic property of $U_{n}$. (We choose the subscripts so that $\left|u_{1}\right|>\left|u_{2}\right|>\cdots>$ $\left.\left|u_{n}\right|.\right)$

(IV) When a solution to an $n$ th-order linear difference equation is known, that solution can be used in conjunction with the equation to derive a new linear difference equation of $(n-1)$ th order [5]. We shall show that if the solution $U_{n}$, found in (III), is used to derive an equation of order $(n-1)$ then this equation has a fundamental system of solutions $U_{i, 1}$ with

$$
\lim _{m \rightarrow \infty} \frac{U_{i, 1}(\omega+m+1)}{U_{i, 1}(\omega+m)}=\frac{u_{i}}{u_{n}}, \quad i=1,2, \cdots,(n-1) .
$$

The $U_{i, 1}$, like the $U_{i}$, have distinct asymptotic properties. Thus we can proceed by steps (I), (II), and (III) to find a solution $U_{n-1,1}$. This solution can then be used to find an equation of order $(n-2)$ and so on.

(V) Finally we get the desired $U_{i}$ from the solution $U_{i, 1}$ by

$$
U_{i}(\omega)=U_{n}(\omega) S_{c}^{\omega}\left[U_{i, 1}(X)\right] \Delta X,
$$

where $S_{c}^{\omega}$ [ ] $\Delta X$ is Norlund's summing operator [6]. The various steps of the algorithm are repeated until all the desired $U_{i}$ are found.

Formally the algorithm may be written as follows: 
(I) Let

$$
U^{i}(\omega)= \begin{cases}\omega^{i-1} & \text { for } 0 \leq \operatorname{Re}[\omega]<n \\ -\left[a_{n-1}(\omega-n) U^{i}(\omega-1)+a_{n-2}(\omega-n) U^{i}(\omega-2)\right. & +\ldots \\ \left.+a_{0}(\omega-n) U^{i}(\omega-n)\right] & \text { recursively first } \\ & \text { for } n \leq \operatorname{Re}[\omega]<(n+1) \\ & \text { then } \\ & (n+1) \leq \operatorname{Re}[\omega]<(n+2) \\ \frac{1}{a_{0}(\omega)}\left[U^{i}(\omega+n)+a_{n-1}(\omega) U^{i}(\omega+n-1)\right. & \text { for all } \\ \left.+a_{1}(\omega) U^{i}(\omega+1)\right] & \text { Re }[\omega] \geq n \\ & \text { recursively first } \\ & \text { for } 0>\operatorname{Re}[\omega] \geq-1\end{cases}
$$

Justification.

A) The $U^{i}$ are well defined solutions by condition (2b) and Ref. [5].

B) The $U^{i}$ form a fundamental system of solutions because

a) for $0 \leq \operatorname{Re}[\omega]<1$, Casorati's determinant,

$$
D(\omega)=\left|\begin{array}{cccc}
1 & \omega & & \omega^{n-1} \\
1 & \omega+1 & & (\omega+1)^{n-1} \\
\vdots & & & \vdots \\
1 & \omega+n-1 & \cdots & (\omega+n-1)^{n-1}
\end{array}\right|
$$

is a Vandermond determinant [7], which is easily seen to be non zero.

b) By Heymann's theorem [8]

$$
D(\omega+1)=(-1)^{n} a_{0}(\omega) D(\omega) .
$$

c) By Condition (2b) above $D(\omega) \neq 0$ for all $\omega$.

d) By Casorati's theorem ${ }^{9}$

$D(\omega) \neq 0 \Rightarrow$ the $U^{i}$, sorm a fundamental system.

C) The $U^{i}$ s can be written formally as

$$
\begin{aligned}
& U^{1}=p_{1}^{1} U_{1}+p_{2}^{1} U_{2}+\cdots+p_{n}^{1} U_{n}, \\
& U^{2}=p^{2}\left[p_{1}^{1} U_{1}+p_{2}^{2} U_{2}+\cdots+p_{n}^{2} U_{n}\right], \\
& U^{3}=p^{3}\left[p_{1}^{1} U_{1}+p_{2}^{3} U_{2}+\cdots+p_{n}^{3} U_{n}\right] \\
& \vdots \\
& U^{n}=p^{n}\left[p_{1}^{1} U_{1}+p_{2}^{n} U_{2}+\cdots+p_{n}^{n} U_{n}\right],
\end{aligned}
$$


where the $p^{i}$ 's and $p_{i}^{i}$ 's are periodic in $\operatorname{Re}[\omega]$ with period one. The $u_{i}^{\text {'s }}$ are ordered so that

$$
\left|u_{1}\right|>\left|u_{2}\right|>\cdots>\left|u_{n}\right| \text {. }
$$

(II) If $U^{1}(\omega+m) \neq 0$ let

$$
p^{i}(\omega, m)=\frac{U^{i}(\omega+m)}{U^{1}(\omega+m)}
$$

Justification.

If $p_{1}^{1}(\omega) \neq 0$ then

$$
\lim _{m \rightarrow \infty} p^{i}(\omega, m)=p^{i}(\omega)
$$

To show this we note

a) By (3) for every $\epsilon>0 \exists M_{i}(\omega)$ and a real, positive $K_{i}(\omega) \ni$ for $m<M_{\text {, }}(\omega)$

$$
K_{i}(\omega)\left(\left|u_{i}\right|-\epsilon\right)^{m-M_{i}(\omega)}<\left|U_{i}(\omega+m)\right|<K_{i}(\omega)\left(\left|u_{i}\right|+\epsilon\right)^{m-M_{i}(\omega)} .
$$

b) For

$$
\epsilon<\frac{\left|u_{1}\right|-\left|u_{2}\right|}{2}
$$

and $m>\max M_{i}=M_{0}$ we have

$$
\left|\frac{U_{i}(\omega+n)}{U_{1}(\omega+m)}\right|<\frac{K_{i}(\omega)}{K_{1}(\omega)} \frac{\left(\left|u_{i}\right|+\epsilon\right)^{M_{0}-M_{i}}}{\left(\left|u_{1}\right|-\epsilon\right)^{M_{0}-M_{1}}}\left[\frac{\left|u_{i}\right|+\epsilon}{\left|u_{1}\right|-\epsilon}\right]^{m-M_{\bullet}} .
$$

So for $j \neq 1$ and $K_{1}(\omega) \neq 0$ we have

$$
\lim _{m \rightarrow \infty} \frac{U_{i}(\omega+m)}{U_{1}(\omega+m)}=0 .
$$

c) The rest is straightforward.

(III) Let

$$
U^{i, 1}=\frac{U^{i}}{p^{i}}-U^{1} \quad \text { for } \quad i=2,3, \cdots, n
$$

Justification.

The $U^{i, 1}$ can be written formally as

$$
\begin{aligned}
& U^{2,1}=p_{2}^{2,1} U_{2}+p_{3}^{2,1} U_{3}+\cdots+p_{n}^{2,1} U_{n}, \\
& U^{3,1}=p^{3,1}\left[p_{2}^{2,1} U_{2}+p_{2}^{3,1} U_{3}+\cdots+p_{n}^{3,1} U_{n}\right], \\
& \vdots \\
& U^{n, 1}=p^{n, 1}\left[p_{2}^{2,1} U_{2}+p_{3}^{n, 1} U_{3}+\cdots+p_{n}^{n, 1} U_{n}\right],
\end{aligned}
$$

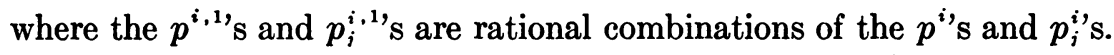

This form is the same as I(c), so steps (II) and (III) can be repeated until there remains only

$$
U^{n, n-1}=p_{n}^{n, n-1} U_{n} .
$$

This solution is a member of Perron's fundamental system relative to the root $u_{n}$. Henceforth we suppress the superscripts and refer to this solution as $U_{n}$. 
(IV) Consider the equation

$$
U_{, 1}(\omega+n-1)+a_{n-2,1}(\omega) U_{, 1}(\omega+n-2)+\cdots+a_{0,1}(\omega) U_{, 1}(\omega)=0
$$

with

$$
-a_{s, 1}(\omega)=a_{0}(\omega) U_{n}(\omega)+\cdots+a_{s}(\omega) U_{n}(\omega+s) .
$$

Justification .

A) Equation (4) has a fundamental system of solutions [5]

$$
U_{i, 1}=\Delta \frac{U_{i}}{U_{n}}, \quad i=1,2, \cdots,(n-1),
$$

where

$$
\Delta f(X)=f(X+1)-f(X) .
$$

B) $\quad \lim _{m \rightarrow \infty} \frac{U_{i, 1}(\omega+n+1)}{U_{i, 1}(\omega+m)}=\frac{u_{i}}{u_{n}}$.

To show this we write

$$
\begin{aligned}
\frac{U_{i, 1}(\omega+m+1)}{U_{i, 1}(\omega+m)}= & {\left[\frac{U_{i}(\omega+m+2)}{U_{n}(\omega+m+2)}-\frac{U_{i}(\omega+m+1)}{U_{n}(\omega+m+1)}\right] } \\
= & \left\{\left[\frac{U_{i}(\omega+m+2)}{U_{i}(\omega+m)}\right] /\left[\frac{U_{i}(\omega+m+1)}{U_{n}(\omega+m+1)}-\frac{U_{i}(\omega+m)}{U_{n}(\omega+m)}\right]^{-1},\right. \\
& -\left[\frac{U_{n}(\omega+m+1)}{U_{n}(\omega+m)}\right] \\
& \cdot\left\{\left(\left[\frac{U_{i}(\omega+m)}{U_{i}(\omega+m)}\right] /\left[\frac{U_{n}(\omega+m+1)}{U_{n}(\omega+m)}\right]\right\}\right. \\
&
\end{aligned}
$$

Thus

$$
\lim _{m \rightarrow \infty} \frac{U_{i, 1}(\omega+m+1)}{U_{i, 1}(\omega+m)}=\frac{\left(u_{i}^{2} / u_{n}^{2}\right)-\left(u_{i} / u_{n}\right)}{\left(u_{i} / u_{n}\right)-1}=\frac{u_{i}}{u_{n}} .
$$

C) The construction procedure (I) gives a fundamental system of solutions to Eq. (4). By Heymann's theorem the only zeros of $D(\omega)$ are congruent to the zeros of $a_{0,1}$, but these are considered singular points of the equation. Thus the hypotheses of Casorati's theorem are satisfied.

D) Repeated application of (II) and (III) leads to a solution with the asymptotic property of $U_{(n-1), 1}$. This solution, along with Eq. (4), can be used to construct an equation of order $(n-2)$ with the same properties as Eq. (4).

(V) Let

$$
U_{i}=U_{n} S_{c}^{\omega}\left[U_{i, 1}(X)\right] \Delta X,
$$

where $\int_{c}^{\omega}[$ [ ] $\Delta X$ is Norlund's summing operator [6]. 


\section{Justification.}

These $U_{i}$ are the solutions $U_{i}$ to Eq. (1) that we desire because $\int_{c}^{\omega}$ is the inverse of $\Delta$ (step (IV)A) to within a periodic function. Thus the problem is solved.

Discussion. In the various steps of the algorithm divisions are required. For these operations we must be sure that the divisor is non zero. First we note that if the coefficient of Eq. (1) are analytic along a line parallel to the real $\omega$ axis except for isolated singularities, then the solutions $U^{i}$ generated in step (I) will have the same property along that line except at the integer values of $\operatorname{Re}[\omega]$. Therefore the zeros of the various divisors will be discrete, and the algorithm can fail because of attempted division by zero only at a discrete set of points.

The other place where the algorithm as stated may fail is in step (II) where we require $p_{1}^{1}(\omega) \neq 0$. It is possible that our construction of the $U^{i}$ gives a $U^{1}$ which is independent of $U_{1}$. Since the $U^{i}$ form a fundamental system, at least one of the $U^{i}$ must depend on $U_{1}$. Therefore if we find that the $p^{i}(\omega, n)$ do not tend to a limit, we must renumber the $U^{i}$ so that the new $U^{1}$ is one of the $U^{i}$ that depends on $U_{1}$. Throughout the elimination process there is the possibility of this analogous situation occurring. However, it can always be circumvented by renumbering the $U^{\prime}$.

In the parametric network problem there are symmetries in the original homogeneous equation. These symmetries can be used to reduce the number of operations required in the algorithm. In a subsequent paper these symmetries and the corresponding reductions will be discussed.

Acknowledgement. The author wishes to express his thanks for the helpful suggestions given by Dr. D. R. Anderson of the Hughes Research Laboratories.

\section{REFERENCES}

1. M. A. Yegrafov, A new proof of a theorem of Perron, Proc. Acad. Sci. USSR Math. Ser. 17, 77-82 (1953)

2. B. J. Leon, A Frequency-domain theory for parametric networks, IRE Trans., CT-7, 321-329 (1960)

3. L. M. Milne-Thomson, The calculus of finite differences, Macmillan Co., Ltd., London, 1933; reprint St. Martin's Press, New York, 1951

4. L. M. Milne-Thomson, op. cit., Sec. 12.01

5. L. M. Milne-Thomson, op. cit., Sec. 12.3

6. L. M. Milne-Thomson, op. cit., Sec. 8.0

7. L. M. Milne-Thomson, op. cit., Sec. 1.5

8. L. M. Milne-Thomson, op. cit., Sec. 12.12

9. L. M. Milne-Thomson, op. cit., Sec. 12.11 